\title{
Application of Vendor-Neutral Iterative Reconstruction Technique to Pediatric Abdominal Computed Tomography
}

\author{
Woo Hyeon Lim, $M D^{1,2}$, Young Hun Choi, $M D^{1,2}$, Ji Eun Park, $M D^{1}$, Yeon Jin Cho, $M D^{1,2}$, \\ Seunghyun Lee, $M D^{1,2}$, Jung-Eun Cheon, $M D$, $P h D^{1,2,3}$, Woo Sun Kim, MD, PhD ${ }^{1,2,3}$, \\ In-One Kim, MD, PhD ${ }^{1,2,3}$, Jong Hyo Kim, MD, $\mathrm{PhD}^{2,4,5}$
}

${ }^{1}$ Department of Radiology, Seoul National University Hospital, Seoul, Korea; ${ }^{2}$ Department of Radiology, Seoul National University College of Medicine, Seoul, Korea; ${ }^{3}$ Institute of Radiation Medicine, Seoul National University Medical Research Center, Seoul, Korea; ${ }^{4}$ Department of Transdisciplinary Studies, Graduate School of Convergence Science and Technology, Seoul National University, Seoul, Korea; ${ }^{5}$ Advanced Institute of Convergence Technology, Suwon, Korea

Objective: To compare image qualities between vendor-neutral and vendor-specific hybrid iterative reconstruction (IR) techniques for abdominopelvic computed tomography (CT) in young patients.

Materials and Methods: In phantom study, we used an anthropomorphic pediatric phantom, age-equivalent to 5-year-old, and reconstructed $\mathrm{CT}$ data using traditional filtered back projection (FBP), vendor-specific and vendor-neutral IR techniques (ClariCT; ClariPI) in various radiation doses. Noise, low-contrast detectability and subjective spatial resolution were compared between FBP, vendor-specific (i.e., iDose1 to 5; Philips Healthcare), and vendor-neutral (i.e., ClariCT1 to 5) IR techniques in phantom. In 43 patients (median, 14 years; age range 1-19 years), noise, contrast-to-noise ratio (CNR), and qualitative image quality scores of abdominopelvic CT were compared between FBP, iDose level 4 (iDose4), and ClariCT level 2 (ClariCT2), which showed most similar image quality to clinically used vendor-specific IR images (i.e., iDose4) in phantom study. Noise, CNR, and qualitative imaging scores were compared using one-way repeated measure analysis of variance.

Results: In phantom study, ClariCT2 showed noise level similar to iDose4 (14.68-7.66 Hounsfield unit [HU] vs. 14.78-6.99 HU at CT dose index volume range of 0.8-3.8 mGy). Subjective low-contrast detectability and spatial resolution were similar between ClariCT2 and iDose4. In clinical study, ClariCT2 was equivalent to iDose4 for noise (14.26-17.33 vs. 16.01-18.90) and CNR (3.55-5.24 vs. 3.20-4.60) $(p>0.05)$. For qualitative imaging scores, the overall image quality ([reader 1 , reader 2]; 2.74 vs. $2.07,3.02$ vs. 2.28$)$ and noise (2.88 vs. $2.23,2.93$ vs. 2.33$)$ of ClariCT2 were superior to those of $\operatorname{FBP}(p<0.05)$, and not different from those of iDose4 (2.74 vs. $2.72,3.02$ vs. $2.98 ; 2.88$ vs. $2.77,2.93$ vs. 2.86$)(p>0.05)$.

Conclusion: Vendor-neutral IR technique shows image quality similar to that of clinically used vendor-specific hybrid IR technique for abdominopelvic CT in young patients.

Keywords: Iterative reconstruction; Pediatric; Computed tomography; Abdomen; Vendor-neutral; Phantom

\section{INTRODUCTION}

Iterative reconstruction (IR) technique is an important radiation dose reduction strategy and could achieve radiation dose reduction without significant compromise of image quality, compared with filtered back projection (FBP) of routine radiation dose (1-7). The iterative noise reduction process can be performed in different domains (in image domain alone, sinogram domain alone, or both domains) depending on IR techniques $(7,8)$. Computed tomography

Received October 12, 2018; accepted after revision June 5, 2019.

This study was supported by a grant of the Korea Health Technology R\&D Project through the Korea Health Industry Development Institute (KHIDI), funded by the Ministry of Health and Welfare, Republic of Korea (grant number: HI15C1532), and a grant of the Institute for Information \& communications Technology Promotion (IITP), funded by the Korea government (MSIP)(No.2017-0-01329). Corresponding author: Young Hun Choi, MD, Department of Radiology, Seoul National University Hospital, 101 Daehak-ro, Jongno-gu, Seoul 03080, Korea.

- Tel: (822) 2072-3600 • Fax: (822) 747-5781•E-mail: iater@snu.ac.kr

This is an 0pen Access article distributed under the terms of the Creative Commons Attribution Non-Commercial License (https:// creativecommons.org/licenses/by-nc/4.0) which permits unrestricted non-commercial use, distribution, and reproduction in any medium, provided the original work is properly cited. 
(CT) vendors have adopted different algorithms according to their application choices and development stages, which led to a variety of vendor-specific IR techniques in practice. Hybrid IR, which uses the relevant information in both the sinogram and image domains, is the most widely used technique in vendors' IR packages today because of its superior performance and computational efficiency (7).

However, using individual vendor-specific IR techniques in a setting of multiple CT units from different vendors may be more expensive than using vendor-neutral IR packages (3). Another critical problem is unavailability of vendor-specific IR techniques compatible with outdated CT machines in current operation. In this situation, vendorneutral IR techniques can be a good solution to decrease radiation exposure without compromising daily workflow, if vendor-neutral IR techniques are comparable to vendorspecific techniques.

Its applicability would be greater in young patients, because radiation dose reduction is a major issue in these patients due to their vulnerability to radiation $(9,10)$. However, to the best of our knowledge, no study has compared the image quality between vendor-neutral and vendor-specific IR techniques in young patients.

ClariCT (ClariPI, Seoul, Korea) is a commercially available vendor-neutral IR technique, which features digital imaging and communications in medicine (DICOM)-based hybrid IR. ClariCT shares the same procedures of the hybrid IR as used in vendor-specific hybrid IR techniques with the advantage of superior noise reduction performance compared with image-based techniques: reduction of sinogram specific noises that are more easily captured in sinogram-domain such as salient local variations of attenuation coefficients on highly attenuated ray paths, followed by additional enhancement steps in image-domain such as edge-adaptive blending of image sub-components. Unlike the vendorspecific hybrid IR, ClariCT includes an additional step of sinogram synthesis from DICOM CT image which is not required in vendor-specific IR techniques. A sophisticated sinogram synthesis procedure including the use of appropriate scanner geometry and advanced forward and backward projection techniques is regarded the key element enabling its denoising performance to potentially match with the vendor-specific hybrid IR techniques $(11,12)$. Information about scanner geometry was obtained from both DICOM header and open sources such as "http://www. impactsca.org/." Thus, ClaricT may be unique in potentially providing enhanced denoising performance and convenient vendor-neutral technique.

This study aimed to evaluate the image quality of the vendor-neutral IR technique and compare it with that of FBP and vendor-specific hybrid IR technique in abdominopelvic CT for young patients through phantom and clinical studies.

\section{MATERIALS AND METHODS}

Our Institutional Review Board (IRB) approved this retrospective study and waived the requirement for informed consent.

\section{Phantom}

In the phantom study, a 5-year-old age-equivalent anthropomorphic pediatric phantom (ATOM model 705; CIRS, Norfolk, VA, USA) and a CT imaging quality assurance kit (Model 700-QA; CIRS) made of tissue equivalent epoxy resins were used. The $\mathrm{CT}$ image quality assurance kit included two soft-tissue inserts of cylindrical and line pair targets; they were positioned in the upper abdomen of the pediatric phantom (Fig. 1). The height and axial dimension of the pediatric phantom was $110 \mathrm{~cm}$ and $17.0 \times 14.0 \mathrm{~cm}$ at the level of the upper abdomen, respectively. The calculated effective diameter was $15.4 \mathrm{~cm}$ (13-15). Details of phantom and soft-tissue inserts are follows: 1 ) The soft-tissue insert with cylindrical targets is composed of 18 low-contrast targets (with 6 variable sizes, ranging from $1.2 \mathrm{~mm}$ to 7 $\mathrm{mm}$ ) with 20 Hounsfield unit (HU) contrast higher than the background (16). 2) The soft-tissue insert with line pair targets is made up of five targets $(6,8,10,11$, and 12 line pairs per centimeter) of which the attenuation (HU) is 300 HU higher than the background (16).

The pediatric phantom was scanned through the 256-slice CT scanner (iCT 256; Philips Healthcare, Andover, MA, USA) with an effective tube current-time product of 19, 24, 47, and $93 \mathrm{mAs}$ at a kilovoltage of $100 \mathrm{kVp}$. The volume CT dose index $\left(\mathrm{CTDI}_{\text {vol }}\right)$ was $0.8,1.0,1.9$, and $3.8 \mathrm{mGy}$, respectively. The other scanning parameters were as follows: detector collimation: $0.625 \times 40 \mathrm{~mm}$, gantry rotation time: 0.27 seconds, and pitch: 0.617 . Automatic tube current modulation was not applied for the phantom study.

The CT images were reconstructed for each scan using traditional FBP and vendor-specific IR (iDose; Philips Healthcare) with five different IR strengths. FBP and iDose images were reconstructed using a standard routine body reconstruction filter (B filter; Philips Healthcare). 


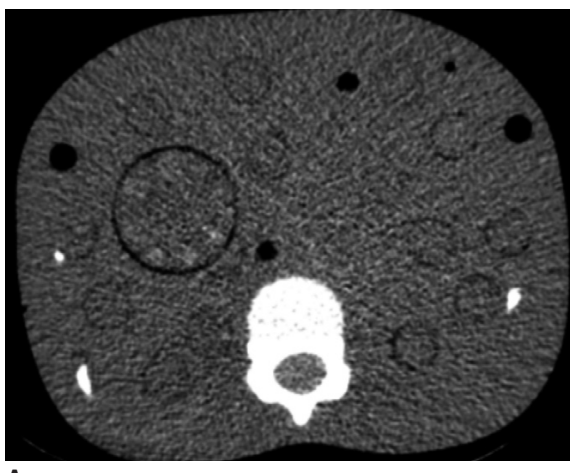

A

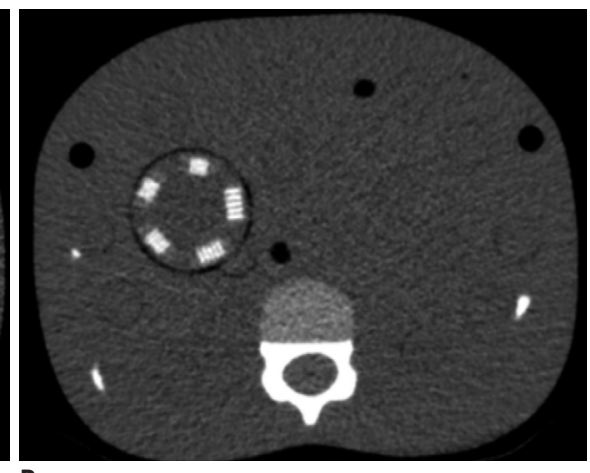

B

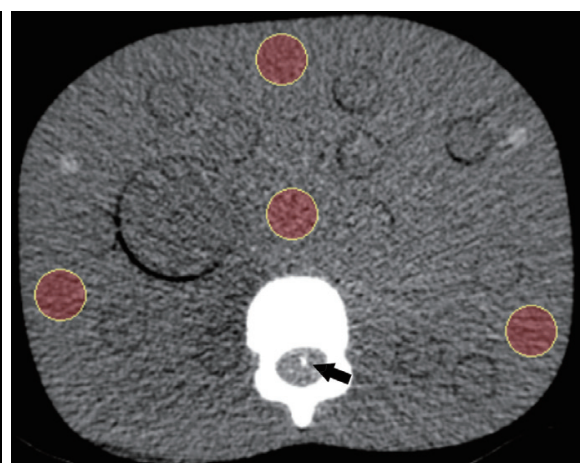

C

Fig. 1. CT images of pediatric anthropomorphic phantom.

Axial images obtained at level of upper abdomen with cylindrical (A) and line pair targets (B) inserted. Both images were obtained at CTDI $_{\text {vol }}$ level of 1.9 mGy and reconstructed using FBP. A. Window width: 200 and level: 50. B. Window width: 500 and level: 100. C. For quantitative noise measurements, four ROIs were drawn on each image: one ROI was located at center, and three were at peripheral portion of phantom. Wire located in spinal canal (arrow) was used for MTF measurement. CT = computed tomography, CTDI $_{\text {vol }}=$ volume CT dose index, FBP $=$ filtered back projection, MTF = modulation transfer function, $\mathrm{ROI}=$ region of interest

Images were also reconstructed using vendor-neutral reconstruction technique (ClariCT) with five different levels of reconstruction strength from the FBP images. The other image reconstruction parameters were the same throughout: display field of view, $220 \times 220 \mathrm{~mm}$; image matrix, $512 \times$ 512; slice thickness, $3 \mathrm{~mm}$; and slice overlap, $1 \mathrm{~mm}$. For the ClaricT, mean denoising time per slice was 0.3 seconds, and overall transfer and processing time of an exam was 2-3 minutes.

For quantitative analyses of image noise, a single radiologist (with 3 years' experience with $\mathrm{CT}$ ) selected one image of the same level at the upper abdomen from each of the reconstructions at different radiation dose levels. Four regions of interest (ROIs) were drawn on each image (Fig. 1) at the picture archiving and communication system (PACS) workstation (Infinitt; Infinitt Healthcare, Seoul, Korea) with displays calibrated to the DICOM grayscale standard display function. One ROI was located at the center of the phantom, and three were at the peripheral portion of the phantom, as previously reported (15). The ROI area was constant at $200 \mathrm{~mm}^{2}$. The image noise for each reconstruction series was the mean of four standard deviation (SD) values measured from four ROIs.

In the quantitative analyses of spatial resolution, images obtained at the CTDI $_{\text {vol }}$ of 1.9 mGy were used. Modulation transfer function (MTF) value was obtained using the wire located in the spinal canal (Fig. 1) with in-house software based on the method reported by Nickoloff (17). Image pixels within an ROI on the wire were subtracted from the ROI-mean, followed by Fourier transform and radial sampling. ROI size of 16 pixels was used to reduce noise effect (17). The spatial frequency at 10\% MTF $\left(\mathrm{MTF}_{10}\right)$ was used to assess the spatial resolution limit.

Noise power spectrum (NPS) was also evaluated to determine the noise content of images using an in-house software based on the method described by Baek and Pelc (18). Image pixels on an ROI of 32-pixel size placed on a uniform central area were subtracted from the ROImean, followed by Fourier transform, square function of the magnitude component, and normalization with the ROI area (18).

In the qualitative analyses of imaging parameters, two radiologists (with 3 and 13 years' experience with CT, respectively) reviewed images obtained at the CTDI $_{\text {vol }}$ level of $1.9 \mathrm{mGy}$, in consensus, on the same PACS workstation. The cylindrical target was used for evaluation of lowcontrast resolution. In the optimized liver setting (window width, $150 \mathrm{HU}$; window level, $50 \mathrm{HU}$ ) (19), the number of discernible targets and that of sharply defined cylindrical targets were evaluated for low-contrast resolution (20). Using the line pair target, assessment of subjective spatial resolution was performed by counting the number of line pairs, wherein adjacent lines were considered separate lines, with window width of $500 \mathrm{HU}$ and level of $100 \mathrm{HU}$.

\section{Clinical Data}

From August 2015 to April 2016, patients who underwent abdominopelvic CT through multi-detector CT (iCT 256) with available both FBP and iDose reconstruction images were included in the study. A total of 43 young patients (26 males and 17 females; median age, 14 years; age range, 1-19 years) were included. The mean effective diameter at 
the superior mesenteric artery os level was $21.0 \pm 4.6 \mathrm{~cm}$, ranging from 13.1 to $33.2 \mathrm{~cm}$ (13). The clinical indications for abdominal CT and clinically relevant positive findings are described in Supplementary Table 1.

The median CTDI $_{\text {vol }}$ at $32 \mathrm{~cm}$ was $1.7 \mathrm{mGy}$ (range, 0.6-10.7 $\mathrm{mGy}$ ), and $\mathrm{kVp}$ was ranging from 80 to $140 \mathrm{kVp}$ (10 studies using $80 \mathrm{kVp}, 31$ using $100 \mathrm{kVp}, 1$ using $120 \mathrm{kVp}$, and 1 using $140 \mathrm{kVp}$ ). Automated tube current modulation was adapted. Slice thickness was $3 \mathrm{~mm}$, detector collimation was $0.625 \times 40 \mathrm{~mm}$, pitch was 0.617 , gantry rotation time was 0.27 seconds, and Dose Right Index, which is a discrete parameter designed to make a consistent image quality for every patient, was 14.

In our institution, all iDose images were reconstructed at strength level 4 (iDose4), because CT images with iDose4 were thought to be optimal level considering both image qualities and noise, as Karmazyn et al. (21) described.

In quantitative and qualitative comparisons between vendor-neutral and vendor-specific IR techniques, ClaricT images were reconstructed from the FBP images, at the denoising level that was similar to that of clinically used vendor-specific IR images (i.e., iDose4), based on the phantom study results.

For quantitative analysis, noise and contrast-to-noise ratio (CNR) were calculated by drawing ROIs in the liver (four ROIs; mean area, $254.1 \mathrm{~mm}^{2}$; range, 187.3-311.34 $\mathrm{mm}^{2}$ ), pancreas (three ROIs; mean area, $157.3 \mathrm{~mm}^{2}$; range, 68.4-221.6 $\mathrm{mm}^{2}$ ), spleen (two ROIs; mean area, $246.3 \mathrm{~mm}^{2}$; range, 129.9-343.2 $\mathrm{mm}^{2}$ ), and two psoas muscles (single ROI; mean area, $234.1 \mathrm{~mm}^{2}$; range, $154.3-338.8 \mathrm{~mm}^{2}$ ) on FBP, iDose, and ClariCT images by a single radiologist (Supplementary Fig. 1). Large vessels, pancreatic ducts, macroscopic fat infiltration, and focal lesions were carefully avoided. Mean attenuation (HU) and SD of each organ were calculated.

The CNRs of the liver, pancreas, and spleen, relative to the muscle were calculated using the following equations: $C N R=\left(m H U_{0}-m H U_{m}\right) / S_{m}$, where $m H U_{0}$ is the mean attenuation in the organ of interest, $\mathrm{mHU}_{\mathrm{m}}$ is the mean attenuation in the paraspinal muscles, and $\mathrm{SD}_{\mathrm{m}}$ is the image noise of the paraspinal muscle (22).

For qualitative comparisons of imaging parameters, overall image quality, lesion conspicuity, image noise, and image artifact such as streak and beam hardening artifacts were evaluated by two radiologists (Table 1 ). Overall image quality was scored using a 5-point Likert scale (23). Lesion conspicuity was evaluated in 34 patients with lesions over $5 \mathrm{~mm}$ using a 5-point scale (24). The details of lesions in these 34 patients are described in Supplementary Table 2. Image noise and artifact were also rated (25).

\section{Statistical Analysis}

To evaluate the normal distribution of parameters, D'Agostino-Pearson test for normal distribution was performed. For comparison of quantitative noise, CNR, and qualitative image quality scores, one-way repeated measures ANOVA was performed, followed by a post-hoc test using pairwise comparison with Bonferroni correction provided by statistical software.

Interobserver reliability for subjective scoring was assessed by using linear weighted kappa coefficient graded as follows: none to slight, $<0.20$; fair, $0.21-0.40$; moderate, 0.41-0.60; substantial, 0.61-0.80; and excellent, $>0.80$ (26).

All statistical analyses were performed using MedCalc (Version 15.2; MedCalc Software, Ostend, Belgium). A $p$ value of less than 0.05 was considered statistically significant.

Table 1. Criteria for Subjective Image Quality Assessment

\begin{tabular}{|c|c|c|c|c|c|}
\hline Criteria & 1 & 2 & 3 & 4 & 5 \\
\hline $\begin{array}{l}\text { Overall image } \\
\text { quality }\end{array}$ & $\begin{array}{l}\text { Unacceptable, } \\
\text { no diagnosis possible }\end{array}$ & $\begin{array}{l}\text { Poor, } \\
\text { diagnostic confidence } \\
\text { substantially reduced }\end{array}$ & $\begin{array}{l}\text { Moderate, } \\
\text { sufficient for } \\
\text { diagnosis }\end{array}$ & Good & Excellent \\
\hline Lesion conspicuity & Unable to see & Blurry but visualized & Acceptable & Good & Excellent \\
\hline Image noise & Unacceptable & Definitely noisy & $\begin{array}{l}\text { Slightly noisy, } \\
\text { but acceptable }\end{array}$ & $\begin{array}{l}\text { Minimal noise, } \\
\text { not affecting } \\
\text { diagnostic quality }\end{array}$ & $\begin{array}{l}\text { No perceivable } \\
\text { noise }\end{array}$ \\
\hline Artifact & Severe artifact & $\begin{array}{l}\text { Moderate artifact, } \\
\text { degrading diagnostic } \\
\text { capability }\end{array}$ & $\begin{array}{l}\text { Slight artifact, } \\
\text { not interfering with } \\
\text { diagnosis capability }\end{array}$ & Minimal artifact & $\begin{array}{l}\text { No perceivable } \\
\text { artifact }\end{array}$ \\
\hline
\end{tabular}




\section{RESULTS}

\section{Phantom Study}

Table 2 shows the image noise levels according to reconstruction methods and strengths and the radiation dose levels. ClaricT level 2 (ClariCT2) showed noise level similar to iDose level 4 (iDose4) at CTDI $_{\text {vol }} 0.8 \mathrm{mGy}$ (ClariCT2 vs. iDose4, 14.68 HU vs. $14.78 \mathrm{HU})$ and $1.0 \mathrm{mGy}(13.58 \mathrm{HU}$ vs. $13.35 \mathrm{HU}$ ), while ClariCT3 and ClariCT4 showed noise level similar to iDose4 at CTDI vol $1.9 \mathrm{mGy}(9.20 \mathrm{HU}$ vs. $9.30 \mathrm{HU}$ ) and $3.8 \mathrm{mGy}(6.93 \mathrm{HU}$ vs. $6.99 \mathrm{HU})$, respectively. ClariCT presented greater denoising effects for images obtained with lower radiation dose. For example, the noise of ClariCT2 was $69.1 \%$ of FBP image at CTDI vol $0.8 \mathrm{mGy}$ and $80.7 \%$ of FBP image at CTDI $3.8 \mathrm{mGy}$, respectively.

Figure 2 shows the MTF and NPS curves of representative

Table 2. Image Noises according to Reconstruction Methods and Strengths at Different Radiation Dose Levels Using 5-Year-0ld Age Equivalent Anthropomorphic Pediatric Phantom

\begin{tabular}{|c|c|c|c|c|}
\hline Reconstruction Methods & $\mathrm{CTDI}_{\mathrm{vol}} 0.8$ & $\mathrm{CTDI}_{\mathrm{vol}} 1.0$ & $\mathrm{CTDI}_{\mathrm{vol}} 1.9$ & CTDI $_{\text {vol }} 3.8$ \\
\hline FBP & $21.26\left(100 \%{ }^{*}\right)$ & $18.92(100 \%)$ & $12.87(100 \%)$ & $9.50(100 \%)$ \\
\hline iDose level 1 & $18.32(86.2 \%)$ & $16.58(87.6 \%)$ & $11.50(89.3 \%)$ & $8.56(90.1 \%)$ \\
\hline iDose level 2 & $17.21(81.0 \%)$ & $15.59(82.4 \%)$ & $10.83(84.1 \%)$ & $8.07(85.0 \%)$ \\
\hline iDose level 3 & $16.04(75.5 \%)$ & $14.51(76.7 \%)$ & $10.08(78.3 \%)$ & $7.54(79.4 \%)$ \\
\hline iDose level 4 & $14.78(69.5 \%)$ & $13.35(70.6 \%)$ & $9.30(72.3 \%)$ & $6.99(73.6 \%)$ \\
\hline iDose level 5 & $13.40(63.1 \%)$ & $12.09(63.9 \%)$ & $8.46(65.7 \%)$ & $6.38(67.2 \%)$ \\
\hline ClariCT level 1 & $15.92(74.9 \%)$ & $14.60(77.2 \%)$ & $10.38(80.7 \%)$ & $8.01(84.5 \%)$ \\
\hline ClariCT level 2 & $14.68(69.1 \%)$ & $13.58(71.8 \%)$ & $9.80(76.2 \%)$ & $7.66(80.7 \%)$ \\
\hline ClariCT level 3 & $13.40(63.1 \%)$ & $12.52(66.2 \%)$ & $9.20(71.5 \%)$ & $7.30(76.9 \%)$ \\
\hline ClariCT level 4 & $12.12(57.0 \%)$ & $11.48(60.7 \%)$ & $8.59(66.7 \%)$ & $6.93(73.0 \%)$ \\
\hline ClaricT level 5 & $10.86(51.1 \%)$ & $10.44(55.2 \%)$ & $7.97(61.9 \%)$ & $6.56(69.1 \%)$ \\
\hline
\end{tabular}

Noise was defined as mean of standard deviations at four regions of interest drawn on pediatric phantom. iDose; Philips Healthcare, ClariCT; ClariPI. *Percentage represents relative noise of image with iterative reconstruction to that of FBP at each radiation dose. CTDI $=$ volume $\mathrm{CT}$ dose index, $\mathrm{FBP}=$ filtered back projection

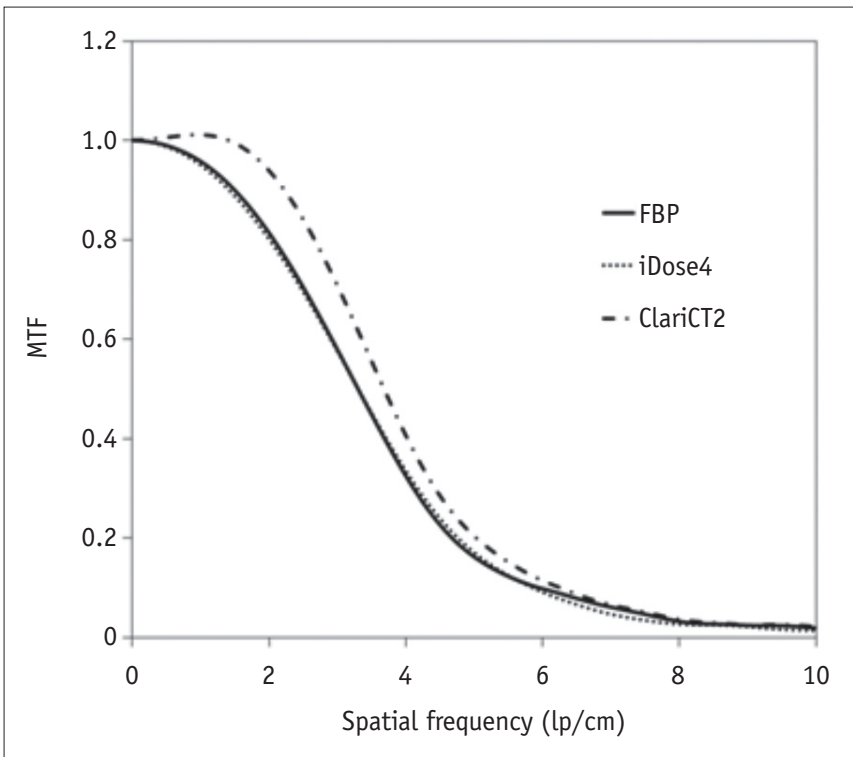

A

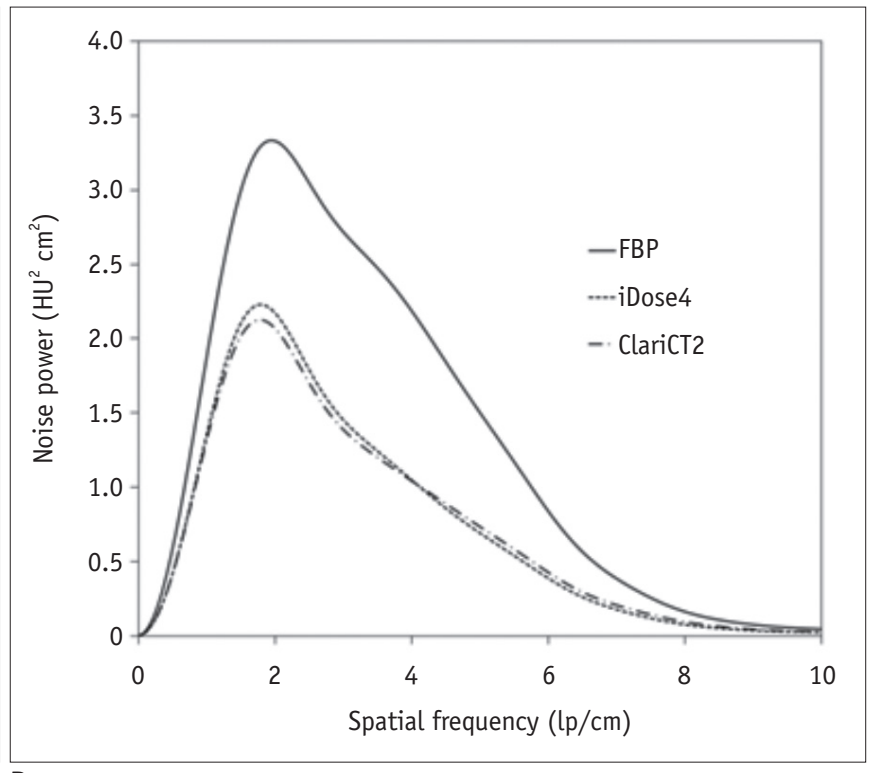

B

Fig. 2. MTF and NPS according to reconstruction methods and strengths.

A. MTF curves of FBP, iDose4, and ClariCT2 are shown. MTF 10 measured at CTDI vol $_{1}$ of 1.9 mGy was $5.93 \mathrm{lp} / \mathrm{cm}$ for FBP, 5.85 for iDose1 to iDose3, and 5.81 for iDose4 and iDose5. In case of ClariCT, MTF ${ }_{10}$ was $6.23,6.23,6.14,6.14$, and $6.19 \mathrm{lp} / \mathrm{cm}$ for ClariCT1 to ClariCT5, respectively. B. Results of NPS according to representative reconstruction methods are shown. Overall, heights of NPS curves of iDose 4 and ClariCT2 are substantially lower than that of FBP, reflecting reduced noise levels. Curve shape in ClariCT2 is flatter than that of iDose4, indicating finer noise texture in ClariCT2 after denoise processing. iDose; Philips Healthcare, ClariCT; ClariPI. ClariCT2 = ClariCT level 2, HU = Hounsfield unit, iDose4 = iDose level $4, \mathrm{MTF}_{10}=10 \% \mathrm{MTF}$, NPS $=$ noise power spectrum 
reconstruction methods, respectively. $\mathrm{MTF}_{10}$ was 5.93, 5.81, and $6.23 \mathrm{lp} / \mathrm{cm}$ for FBP, iDose4, and ClariCT2, respectively. In terms of NPS curve, the curve shape of ClariCT2 was flatter than that of iDose4, suggesting finer noise texture of ClariCT.

Table 3 presents the results of subjective assessments of low-contrast resolution and spatial resolution. With regard to iDose and ClariCT, the number of sharply defined objects increased with increasing reconstruction strengths. The number of line pairs where adjacent lines were separated from each other was three for all three reconstruction methods.

\section{Clinical Data}

ClariCT2 was most similar to clinically used iDose4 in terms of noise and MTF measured in the phantom study. Figures 3 and 4 show representative images of FBP, iDose4, and ClariCT2.

Table 4 describes the results of image noise and CNR. Image noises and CNRs of three reconstruction methods showed

Table 3. Results of Subjective Analysis of Low-Contrast Resolution and Spatial Resolution Using Cylindrical Targets and Line Pairs

\begin{tabular}{lccc}
\hline Reconstruction Methods & Discernable Target & Sharply Demarcated Target & Visually Separated Line Pairs \\
\hline FBP & 8 & 5 & 3 \\
iDose level 1 & 9 & 6 & 3 \\
iDose level 2 & 9 & 7 & 3 \\
iDose level 3 & 9 & 7 & 3 \\
iDose level 4 & 9 & 8 & 3 \\
iDose level 5 & 9 & 8 & 3 \\
ClariCT level 1 & 9 & 7 & 3 \\
ClariCT level 2 & 9 & 7 & 3 \\
ClariCT level 3 & 9 & 8 & 3 \\
ClariCT level 4 & 9 & 8 & 3 \\
ClariCT level 5 & 9 & 8 & 3 \\
\hline
\end{tabular}

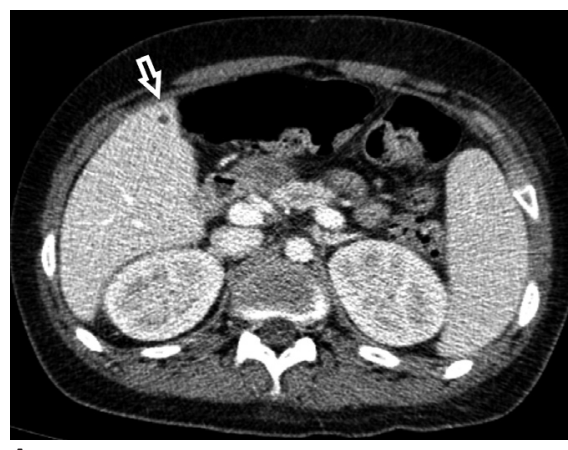

A
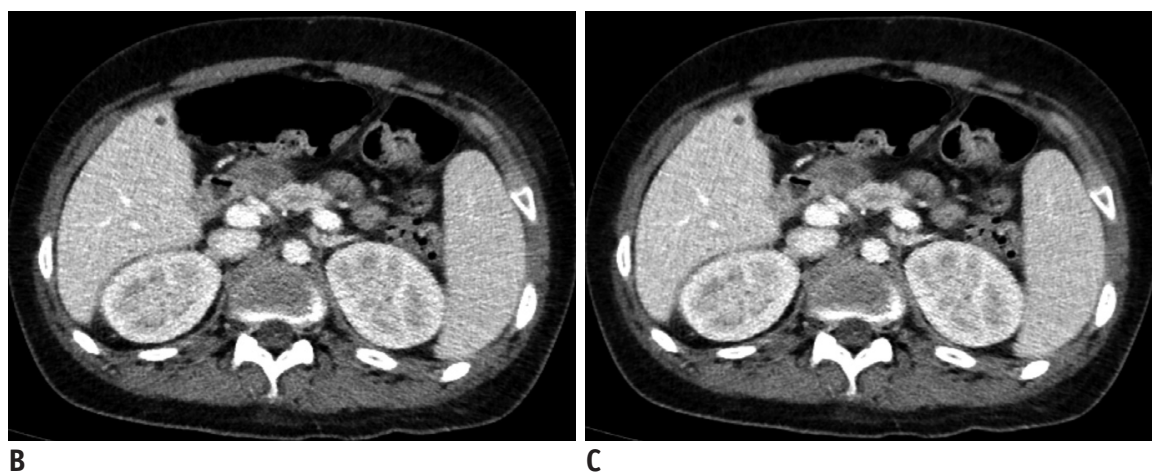

C

Fig. 3. 12-year-old male patient was taken CT scan because of fever.

Abdominal CT scan revealed multifocal fungal abscess in liver (arrow on A) and spleen (not presented). A. FBP. B. iDose4. C. ClariCT2.

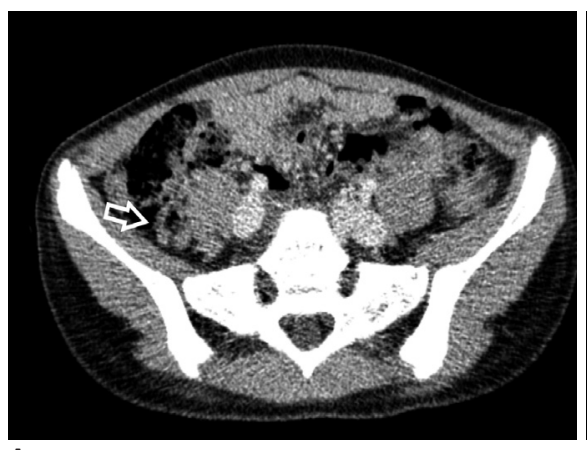

A

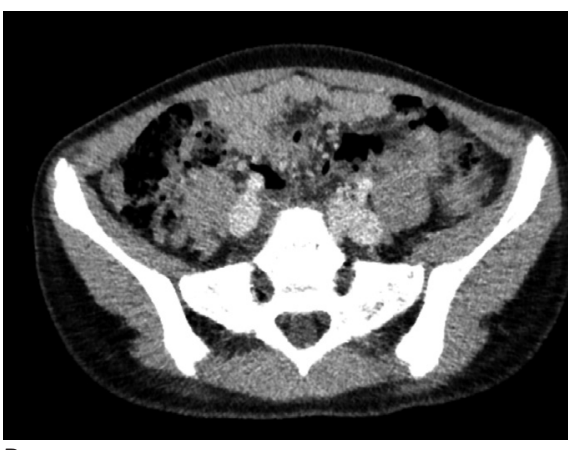

B

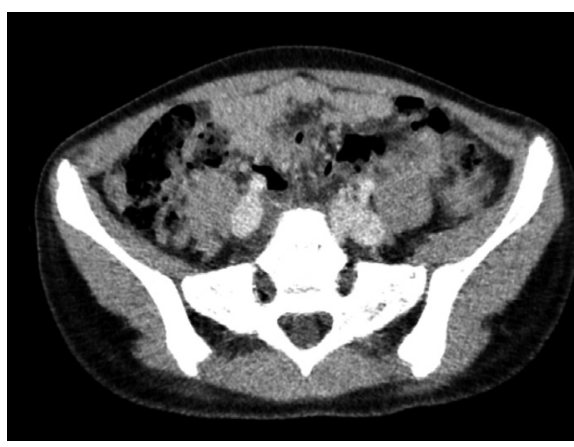

$c$

Fig. 4. 12-year-old female patient visited emergency department with right lower quadrant abdominal pain.

Patient was taken CT scan to rule out acute appendicitis, and CT scan revealed clear appendix (arrow on A). A. FBP. B. iDose4. C. ClariCT2. 
normal distribution. ClariCT2 showed significantly less noise and higher CNR than FBP for all organs (noise, 14.26-17.33 vs. 23.58-27.67; CNR, $3.55-5.24$ vs. $2.25-3.22)(p<0.05)$. The noise and CNR of iDose4 were not significantly different from those of ClariCT2 (noise, $16.01-18.90$ vs. $14.26-17.33$; CNR, $3.20-4.60$ vs. $3.55-5.24)(p>0.05)$.

Table 5 summarizes the subjective image quality scores. Two independent readers evaluated that overall image quality ([reader 1 , reader 2]; 2.74 vs. $2.07,3.02$ vs. 2.28 ) and noise (2.88 vs. $2.23,2.93$ vs. 2.33 ) of ClariCT2 were superior to those of FBP $(p<0.05)$. Similar with quantitative analyses, overall image quality (2.74 vs. 2.72 , 3.02 vs. 2.98 ) and noise (2.88 vs. $2.77,2.93$ vs. 2.86 ) scores were not significantly different between ClariCT2 and iDose $4(p>0.05)$. The artifact and lesion conspicuity scores were similar in all three reconstructions $(p>$ $0.05)$. Interobserver reliability for qualitative scoring was moderate to almost perfect agreement (weighted kappa = 0.456-0.929) between the two observers (Table 5).

\section{DISCUSSION}

ClariCT is a vendor-neutral IR technique which features DICOM-based sinogram synthesis and hybrid IR and has advantage of both sinogram-based and image-based denoising. The hybrid algorithm in ClariCT is performed in a unique arrangement. The first step is forward projection of an FBP-generated CT image to create a synthesized sinogram, which is the same part shared in most hybrid reconstruction techniques. Here, the geometry of the CT system of interest is derived indirectly from the DICOM header and related literature. In the second step, the algorithm analyzes the synthesized sinogram and identifies the noisiest part of sinogram (photon-starved area). This is followed by an extraction of noise sinogram through an iterative process with irregularity and an unusual statistical pattern. Then FBP is applied to the noise sinogram and reconstructs a noise $\mathrm{CT}$ image, which mostly contains streak and irregular directional noises. Subsequently, an additional

Table 4. Image Noise and CNR of Each Organ according to Reconstruction Methods

\begin{tabular}{|c|c|c|c|c|c|}
\hline Parameters & Organs & FBP & iDose4 & ClariCT2 & $P$ \\
\hline \multirow{4}{*}{ Noise } & Liver & $23.58(100 \% *)$ & $16.13(68.4 \%)$ & $14.83(62.9 \%)$ & $<0.001^{\dagger}$ \\
\hline & Pancreas & $27.67(100 \%)$ & $18.90(68.3 \%)$ & $17.33(62.6 \%)$ & $<0.001^{\dagger}$ \\
\hline & Spleen & $25.47(100 \%)$ & $17.23(67.7 \%)$ & $15.63(61.4 \%)$ & $<0.001^{\dagger}$ \\
\hline & Psoas muscle & $23.76(100 \%)$ & $16.01(67.4 \%)$ & $14.26(60.0 \%)$ & $<0.001^{\dagger}$ \\
\hline \multirow{3}{*}{ CNR } & Liver & $2.85(100 \%)$ & $4.09(143.5 \%)$ & $4.64(162.8 \%)$ & $0.012^{\ddagger}$ \\
\hline & Pancreas & $2.25(100 \%)$ & $3.20(142.2 \%)$ & $3.55(157.8 \%)$ & $0.049^{\ddagger}$ \\
\hline & Spleen & $3.22(100 \%)$ & $4.60(142.9 \%)$ & $5.24(162.7 \%)$ & $0.012^{\ddagger}$ \\
\hline
\end{tabular}

*Percentage represents relative value (noise and CNR) of reconstructed images (iDose, ClariCT) to that of FBP, ${ }^{\dagger}$ Post-hoc test revealed mean noise of FBP was significantly higher than those of iDose4 and ClariCT2, ₹Post-hoc test revealed mean CNR of ClariCT2 was significantly higher than that of FBP. CNR = contrast-to-noise ratio, ClariCT2 $=$ ClariCT level 2, iDose $4=$ iDose level 4

Table 5. Subjective Image Quality Scores and Interobserver Reliability for Three Reconstruction Methods

\begin{tabular}{|c|c|c|c|c|c|c|c|}
\hline Imaging Parameters & FBP & $\begin{array}{c}\text { Weighted } \\
\text { Kappa Value }\end{array}$ & iDose4 & $\begin{array}{c}\text { Weighted } \\
\text { Kappa Value }\end{array}$ & ClaricT2 & $\begin{array}{c}\text { Weighted } \\
\text { Kappa Value }\end{array}$ & $P$ \\
\hline \multicolumn{8}{|l|}{ Overall image quality } \\
\hline Reader 1 & 2.07 & \multirow{2}{*}{0.626} & 2.72 & \multirow{2}{*}{0.580} & 2.74 & \multirow{2}{*}{0.559} & $<0.001^{*}$ \\
\hline Reader 2 & 2.28 & & 2.98 & & 3.02 & & $<0.001^{*}$ \\
\hline \multicolumn{8}{|l|}{ Noise } \\
\hline Reader 1 & 2.23 & \multirow{2}{*}{0.622} & 2.77 & \multirow{2}{*}{0.555} & 2.88 & \multirow{2}{*}{0.487} & $<0.001^{*}$ \\
\hline Reader 2 & 2.33 & & 2.86 & & 2.93 & & $0.002^{*}$ \\
\hline \multicolumn{8}{|l|}{ Artifact } \\
\hline Reader 1 & 3.07 & \multirow{2}{*}{0.699} & 3.26 & \multirow{2}{*}{0.456} & 3.26 & \multirow{2}{*}{0.565} & 0.588 \\
\hline Reader 2 & 3.19 & & 3.53 & & 3.49 & & 0.153 \\
\hline \multicolumn{8}{|l|}{ Lesion conspicuity } \\
\hline Reader 1 & 3.68 & \multirow{2}{*}{0.929} & 3.62 & \multirow{2}{*}{0.792} & 3.65 & \multirow{2}{*}{0.824} & 0.962 \\
\hline Reader 2 & 3.79 & & 3.97 & & 3.94 & & 0.645 \\
\hline
\end{tabular}

*In cases of $p$ values $<0.05$, post-hoc tests reveal that mean score of FBP was significantly lower than those of iDose 4 and ClariCT2. 
processing loop is applied in image space to further reduce the noise left after subtraction of the reconstructed noise CT. Finally, the denoised image is adaptively blended with the original FBP image by using the local noise statistic, which attempts to avoid the plastic appearance of the processed image due to excessive noise subtraction. Uniquely in ClariCT, the forward projection and FBP reconstruction steps are performed only using DICOM data; therefore, the entire hybrid IR is carried out in a vendorneutral manner.

We thought that ClariCT may have the potential to provide enhanced denoising performance and radiation dose reduction as well as the convenience of vendorneutral technique. Several previous studies investigated the performance of image-based IR technique and demonstrated similarity with vendor-supplied IR techniques (27-30). However, those studies were conducted in adults; to date, there has been no investigation of image quality using vendor-neutral IR method in young patients.

Previous studies have focused on the comparison of vendor-specific and vendor-neutral IR techniques in low-dose CT (27-30), or the performance evaluation of vendor-neutral IR using homogeneous lesions $(27,30)$. Fletcher et al. (27) reported non-inferiority between vendor-independent and vendor-specific IR methods in CT enterography. Other studies compared image qualities between vendor-specific and vendor-neutral methods (i.e., SafeCT) (28-30), acquiring both low- and standarddose images, prospectively. In our study, the young patients underwent abdominal CT for various reasons, and the radiation doses varied significantly to balance the exposure and diagnostic performance. These parameters may affect the study design and its observations. However, in actual clinical practice, pediatric radiologists examine images of varying qualities depending on the patients' age, radiation dose, or image noise. Additionally, they are required to detect abnormalities of various organs. Evaluation of vendor-neutral IR techniques using multiorgan lesions and varying radiation doses might better represent the actual clinical setting and is advantageous to our study.

Because radiation reduction is a critical issue in young patients, previous studies evaluated the effect of IR at ultralow radiation dose levels with an anthropomorphic phantom $(9,10)$. However, in this study, phantom was not scanned with ultralow radiation dose (less than $0.5 \mathrm{mGy}$ ), because that level of radiation dose may not be used in clinical setting. Indeed, radiation dose was more than 0.5 mGy in our study population.

In terms of image noise reduction, ClaricT showed higher noise reduction power at a lower radiation dose level. This could be of clinical significance especially for young patients, because constant noise level is important for interpretation and is difficult to achieve in those patients due to high variations in body sizes and shapes. This might be attributable to differences in denoise processing between iDose and ClaricT, in which denoising effects on the sinogram and $\mathrm{CT}$ image could be combined differently depending on the confidence of noise separation power in each technique.

MTFs of FBP and iDose were almost identical, while that of ClariCT showed right shift at all spatial frequency ranges leading to a wider area under the curve. NPS curves of ClariCT and iDose showed a stronger downward shift, with a flatter overall shape in ClariCT than seen with iDose. Based on the interpretation of the frequency spectrum, a wider MTF curve represents better capability of preserving details of structural patterns whereas a flatter NPS curve is related to a finer image texture. Therefore, our data may indicate that ClariCT has superior capability than iDose in preserving fine details of structures as well as providing a finer image texture after denoise processing.

In our institute, vendor-provided denoising technique of level 4 (iDose4) has been used, as the images of that level have an acceptable noise level without significant image quality deterioration (21). In the phantom study, absolute noise level of iDose4 was comparable to that of ClariCT2. Based on these phantom study results, ClariCT2 were selected for quality comparison of clinical imaging data.

Indeed, in the subjective analysis, ClaricT2 was comparable to iDose4 in terms of overall image quality and noise. ClariCT2 and iDose4 were also equivalent in artifact and lesion conspicuity scores. However, these two denoising techniques failed to show significant differences in artifact and lesion conspicuity compared with FBP, possibly due to heterogeneity of study population and lesions. The absence of differences in lesion conspicuity could be explained by the fact that evaluation of lesion conspicuity is highly dependent on personal experience and performance, and observer performance may not be directly linked to subjective image quality.

The strength of image-based noise reduction technique is vendor independency. Hence, simultaneous denoise processing of $\mathrm{CT}$ images from multiple vendors is possible 
and can be applied to images obtained with older CT systems for which modern vendor-provided denoising techniques are unavailable. The disadvantage of imagebased denoising is loss of workflow efficiency, as FBP images are transferred to a server, denoised, and then transferred to PACS. In our test environment, the mean denoising time was 0.3 seconds per slice, and the overall transfer and processing of an exam resulted in a 2-3 minutes delay. In many practical applications, however, few minutes delay may not impair clinical workflow. In addition, the use of higher-end workstation may improve the workflow efficiency.

This study has several limitations. First, we evaluated vendor-neutral IR technique in only one type of CT equipment in clinically acceptable range of radiation doses (more than $0.5 \mathrm{mGy}$ ). Therefore, to demonstrate true vendor-neutrality of the technique, evaluation of ClaricT for $\mathrm{CT}$ equipment by other vendors is needed. In addition, we did not compare the performance of ClariCT with modelbased IR techniques. However, in this study, we aimed to evaluate the clinical applicability of ClariCT, which showed more similarity to hybrid IR technique than model-based one, by comparing clinically used hybrid IR technique (i.e., iDose4). Therefore, comparison between ClariCT and other model-based IR technique or evaluation of denoising performance in ultra-low radiation dose (less than 0.5 $\mathrm{mGy)}$ might be required in these contexts. Second, due to limited clinical data, no image reconstruction was made at variable levels other than iDose4; hence, it was difficult to fully compare iDose with ClariCT at all levels. Therefore, we compared the image quality with various levels of reconstruction strength using a pediatric phantom. Third, the objective radiologist performance such as detectability of subtle lesions could not be measured, as those tasks cannot be performed in a fully blinded fashion. Finally, the evaluation of lesion conspicuity according to each disease entity could not be performed, due to the small sample size.

In conclusion, ClariCT is a vendor-neutral IR technique that shows similar image quality to clinically used vendorspecific hybrid IR in pediatric phantom and abdominopelvic $\mathrm{CT}$ in young patients. Additional validation studies using $\mathrm{CT}$ machines from other vendors are required.

\section{Supplementary Materials}

The Data Supplement is available with this article at https://doi.org/10.3348/kjr.2018.0715.

\section{Conflicts of Interest}

Jong Hyo Kim is the inventor of the technique and a share holder of ClariPI inc.

Other authors have no conflict of interest including financial or consultant, institutional and other relationship in this study.

\section{Acknowledgments}

We thank Young Mi Chun, CT clinical scientist manager, Philips Healthcare Korea, for technical support.

\section{ORCID iDs}

Young Hun Choi https://orcid.org/0000-0002-1842-9062

Woo Hyeon Lim https://orcid.org/0000-0001-8985-8473

Ji Eun Park https://orcid.org/0000-0003-1305-0931

Yeon Jin Cho https://orcid.org/0000-0001-9820-3030

Seunghyun Lee https://orcid.org/0000-0003-1858-0640

Jung-Eun Cheon https://orcid.org/0000-0003-1479-2064

Woo Sun Kim https://orcid.org/0000-0003-2184-1311

In-One Kim

https://orcid.org/0000-0001-5800-3536

Jong Hyo Kim

https://orcid.org/0000-0002-5695-4976

\section{REFERENCES}

1. Coakley FV, Gould R, Yeh BM, Arenson RL. CT radiation dose: what can you do right now in your practice? AJR Am J Roentgenol 2011;196:619-625

2. Costello JE, Cecava ND, Tucker JE, Bau JL. CT radiation dose: current controversies and dose reduction strategies. AJR Am J Roentgenol 2013;201:1283-1290

3. Hara AK, Wellnitz CV, Paden RG, Pavlicek W, Sahani DV. Reducing body $C T$ radiation dose: beyond just changing the numbers. AJR Am J Roentgenol 2013;201:33-40

4. Maldjian PD, Goldman AR. Reducing radiation dose in body CT: a primer on dose metrics and key CT technical parameters. AJR Am J Roentgenol 2013;200:741-747

5. Solomon J, Marin D, Roy Choudhury K, Patel B, Samei E. Effect of radiation dose reduction and reconstruction algorithm on image noise, contrast, resolution, and detectability of subtle hypoattenuating liver lesions at multidetector CT: filtered 
back projection versus a commercial model-based iterative reconstruction algorithm. Radiology 2017;284:777-787

6. Zacharias C, Alessio AM, Otto RK, Iyer RS, Philips GS, Swanson J0, et al. Pediatric CT: strategies to lower radiation dose. AJR Am J Roentgenol 2013;200:950-956

7. Willemink MJ, de Jong PA, Leiner T, de Heer LM, Nievelstein RA, Budde RP, et al. Iterative reconstruction techniques for computed tomography Part 1: technical principles. Eur Radiol 2013;23:1623-1631

8. Beister M, Kolditz D, Kalender WA. Iterative reconstruction methods in X-ray CT. Phys Med 2012;28:94-108

9. Lee KB, Goo HW. Quantitative image quality and histogrambased evaluations of an iterative reconstruction algorithm at low-to-ultralow radiation dose levels: a phantom study in chest CT. Korean J Radiol 2018;19:119-129

10. Infante JC, Liu Y, Rigsby CK. CT image quality in sinogram affirmed iterative reconstruction phantom study - is there a point of diminishing returns? Pediatr Radiol 2017;47:333-341

11. Won Kim C, Kim JH. Realistic simulation of reduced-dose CT with noise modeling and sinogram synthesis using DICOM CT images. Med Phys 2014;41:011901

12. Ahn CK, Yang Z, Heo C, Jin H, Park B, Kim JH. A deep learning-enabled iterative reconstruction of ultra-low-dose CT: use of synthetic sinogram-based noise simulation technique. SPIE Medical Imaging 2018;10573:1057335

13. Moore BM, Brady SL, Mirro AE, Kaufman RA. Size-specific dose estimate (SSDE) provides a simple method to calculate organ dose for pediatric CT examinations. Med Phys 2014;41:071917

14. Strauss KJ, Goske MJ. Estimated pediatric radiation dose during CT. Pediatr Radiol 2011;41 Suppl 2:472-482

15. Ryu YJ, Choi YH, Cheon JE, Ha S, Kim WS, Kim IO. Knowledgebased iterative model reconstruction: comparative image quality and radiation dose with a pediatric computed tomography phantom. Pediatr Radiol 2016;46:303-315

16. CIRS. ATOM ${ }^{\circledR}$ Dosimetry Phantoms. Available at: http://www. cirsinc.com/wp-content/uploads/2019/05/701-706-ATOMPB-120418.pdf. Accessed August 9, 2019

17. Nickoloff EL. Measurement of the PSF for a CT scanner: appropriate wire diameter and pixel size. Phys Med Biol 1988;33:149-155

18. Baek J, Pelc NJ. The noise power spectrum in CT with direct fan beam reconstruction. Med Phys 2010;37:2074-2081

19. Mayo-Smith WW, Gupta H, Ridlen MS, Brody JM, Clements NC,
Cronan JJ. Detecting hepatic lesions: the added utility of CT liver window settings. Radiology 1999;210:601-604

20. Löve A, Olsson ML, Siemund R, Stålhammar F, BjörkmanBurtscher IM, Söderberg M. Six iterative reconstruction algorithms in brain CT: a phantom study on image quality at different radiation dose levels. Br J Radiol 2013;86:20130388

21. Karmazyn B, Liang Y, Ai H, Eckert GJ, Cohen MD, Wanner MR, et al. Optimization of hybrid iterative reconstruction level in pediatric body CT. AJR Am J Roentgenol 2014;202:426-431

22. Siegel MJ, Hildebolt C, Bradley D. Effects of automated kilovoltage selection technology on contrast-enhanced pediatric CT and CT angiography. Radiology 2013;268:538-547

23. Ryu YJ, Kim WS, Choi YH, Cheon JE, Lee SM, Cho HH, et al. Pediatric chest CT: wide-volume and helical scan modes in 320-MDCT. AJR Am J Roentgenol 2015;205:1315-1321

24. Lee JH, Choi YH, Cheon JE, Lee SM, Cho HH, Shin SM, et al. Improved abdominal MRI in non-breath-holding children using a radial k-space sampling technique. Pediatr Radiol 2015;45:840-846

25. Russell MT, Fink JR, Rebeles F, Kanal K, Ramos M, Anzai Y. Balancing radiation dose and image quality: clinical applications of neck volume CT. AJNR Am J Neuroradiol 2008;29:727-731

26. McHugh ML. Interrater reliability: the kappa statistic. Biochem Med (Zagreb) 2012;22:276-282

27. Fletcher JG, Hara AK, Fidler JL, Silva AC, Barlow JM, Carter $R E$, et al. Observer performance for adaptive, image-based denoising and filtered back projection compared to scannerbased iterative reconstruction for lower dose CT enterography. Abdom Imaging 2015;40:1050-1059

28. Khawaja RD, Singh S, Blake M, Harisinghani M, Choy G, Karaosmanoglu $A$, et al. Ultralow-dose abdominal computed tomography: comparison of 2 iterative reconstruction techniques in a prospective clinical study. J Comput Assist Tomogr 2015;39:489-498

29. Pourjabbar S, Singh S, Kulkarni N, Muse V, Digumarthy SR, Khawaja RD, et al. Dose reduction for chest CT: comparison of two iterative reconstruction techniques. Acta Radiol 2015;56:688-695

30. Padole A, Digumarthy S, Flores E, Madan R, Mishra S, Sharma $A$, et al. Assessment of chest CT at CTDIvol less than $1 \mathrm{mGy}$ with iterative reconstruction techniques. $\mathrm{Br} \mathrm{J}$ Radiol 2017;90:20160625 